\title{
Some generalizations of Darbo's theorem and applications to fractional integral equations
}

Mohamed Jleli' ${ }^{1}$, Erdal Karapinar ${ }^{2,3}$, Donal O'Regan ${ }^{4}$ and Bessem Samet ${ }^{1 *}$

\section{"Correspondence:}

bsamet@ksu.edu.sa

'Department of Mathematics,

College of Science, King Saud

University, P.O. Box 2455, Riyadh,

11451, Saudi Arabia

Full list of author information is

available at the end of the article

\begin{abstract}
In this paper, some generalizations of Darbo's fixed point theorem are presented. An existence result for a class of fractional integral equations is given as an application of the obtained results.
\end{abstract}

MSC: $47 \mathrm{H} 10 ; 26 \mathrm{~A} 33 ; 45 \mathrm{G} 10$

Keywords: Darbo's theorem; measure of noncompactness; fractional integral equation

\section{Introduction and preliminaries}

Let $E$ be a Banach space over $\mathbb{R}$ (or $\mathbb{C}$ ) with respect to a certain norm $\|\cdot\|$. For any subsets $X$ and $Y$ of $E$, we have the following notations:

$\bar{X}$ denotes the closure of $X$;

$\operatorname{conv}(X)$ denotes the convex hull of $X$;

$P(X)$ denotes the set of nonempty subsets of $X$;

$X+Y$ and $\lambda X(\lambda \in \mathbb{R})$ stand for algebraic operations on sets $X$ and $Y$.

We denote by $\mathcal{B}_{E}$ the family of all nonempty bounded subsets of $E$. Finally, if $X$ is a nonempty subset of $E$ and $T: X \rightarrow X$ is a given operator, we denote by $\operatorname{Fix}(T)$ the set of fixed points of $T$, that is,

$$
\operatorname{Fix}(T)=\{x \in X: T x=x\} .
$$

Banaś and Goebel [1] introduced the following axiomatic definition of the concept of a measure of noncompactness.

Definition 1.1 Let $\sigma: \mathcal{B}_{E} \rightarrow[0, \infty)$ be a given mapping. We say that $\sigma$ is a BG-measure of noncompactness (in the sense of Banaś and Gobel) on $E$ if the following conditions are satisfied:

(i) For every $X \in \mathcal{B}_{E}, \sigma(X)=0$ iff $X$ is precompact.

(ii) For every pair $(X, Y) \in \mathcal{B}_{E} \times \mathcal{B}_{E}$, we have

$$
X \subseteq Y \quad \Longrightarrow \quad \sigma(X) \leq \sigma(Y)
$$

(c) 2016 Jleli et al. This article is distributed under the terms of the Creative Commons Attribution 4.0 International License (http://creativecommons.org/licenses/by/4.0/), which permits unrestricted use, distribution, and reproduction in any medium, provided you give appropriate credit to the original author(s) and the source, provide a link to the Creative Commons license, and indicate if changes were made. 
(iii) For every $X \in \mathcal{B}_{E}$, we have

$$
\sigma(\bar{X})=\sigma(X)=\sigma(\operatorname{conv}(X)) .
$$

(iv) For every pair $(X, Y) \in \mathcal{B}_{E} \times \mathcal{B}_{R}$ and $\lambda \in(0,1)$, we have

$$
\sigma(\lambda X+(1-\lambda) Y) \leq \lambda \sigma(X)+(1-\lambda) \sigma(Y) .
$$

(v) If $\left\{X_{n}\right\} \subseteq \mathcal{B}_{E}$ is a decreasing sequence (w.r.t. $\subseteq$ ) of closed sets such that $\sigma\left(X_{n}\right) \rightarrow 0$ as $n \rightarrow \infty$, then $X_{\infty}:=\bigcap_{n=1}^{\infty} X_{n}$ is nonempty.

Let $\mathcal{X}$ be a nonempty, bounded, closed, and convex subset of the Banach space $E$.

We denote by $\mathcal{D}_{\mathcal{X}}$ the set of self-mappings $D: \mathcal{X} \rightarrow \mathcal{X}$ satisfying the following conditions:

(i) $D$ is a continuous mapping.

(ii) There exist $\sigma: \mathcal{B}_{E} \rightarrow[0, \infty)$, a BG-measure of noncompactness on $E$, and a constant $k \in(0,1)$ such that

$$
\sigma(D W) \leq k \sigma(W), \quad W \in P(\mathcal{X})
$$

The following result is known as Darbo's fixed point theorem (see $[1,2])$.

Theorem 1.2 Let $D: \mathcal{X} \rightarrow \mathcal{X}$ be a mapping that belongs to $\mathcal{D}_{\mathcal{X}}$. Then $D$ has at least one fixed point. Moreover, the set $\operatorname{Fix}(D)$ is precompact.

Many generalizations and extensions of Darbo's fixed point theorem can be found in the literature (see, for example, [3-9] and the references therein). Using the BG-measure of noncompactness, Aghajani et al. [4] obtained the following generalization of Darbo's theorem. Let $\mathcal{F}_{\mathcal{X}}$ be the set of self-mappings $D: \mathcal{X} \rightarrow \mathcal{X}$ satisfying the following conditions:

(i) $D$ is a continuous mapping.

(ii) There exists $\sigma: \mathcal{B}_{E} \rightarrow[0, \infty)$, a BG-measure of noncompactness on $E$, such that for all $\varepsilon>0$, there exists some $\delta_{\varepsilon}>0$ for which

$$
W \in P(\mathcal{X}), \quad \varepsilon \leq \sigma(W)<\varepsilon+\delta_{\varepsilon} \quad \Longrightarrow \quad \sigma(D W)<\varepsilon .
$$

Theorem 1.3 (Aghajani et al. [4]) Let $D: \mathcal{X} \rightarrow \mathcal{X}$ be a mapping that belongs to $\mathcal{F}_{\mathcal{X}}$. Then $D$ has at least one fixed point.

Observe that $\mathcal{D}_{\mathcal{X}} \subseteq \mathcal{F}_{\mathcal{X}}$. In fact, let $D: \mathcal{X} \rightarrow \mathcal{X}$ be a given mapping that belongs to $\mathcal{D}_{\mathcal{X}}$. Let $\varepsilon>0$. From the definition of $\mathcal{D}_{\mathcal{X}}$, there is some $k \in(0,1)$ such that

$$
\sigma(D W) \leq k \sigma(W)
$$

for any nonempty subset $W$ of $\mathcal{X}$. Let $\delta_{\varepsilon}=\left(\frac{1}{k}-1\right) \varepsilon$. Then for any nonempty subset $W$ of $\mathcal{X}$, we have

$$
\varepsilon \leq \sigma(W)<\varepsilon+\delta_{\varepsilon}=\frac{\varepsilon}{k} \quad \Longrightarrow \quad \sigma(D W) \leq k \sigma(W)<\varepsilon,
$$

so $D \in \mathcal{F}_{\mathcal{X}}$. 
In [6], Dhage introduced the following axiomatic definition of the measure of noncompactness.

Definition 1.4 Let $\sigma: \mathcal{B}_{E} \rightarrow[0, \infty)$ be a given mapping. We say that $\sigma$ is a D-measure of noncompactness (in the sense of Dhage) on $E$ if the following conditions are satisfied:

(i) For every $X \in \mathcal{B}_{E}, \sigma(X)=0$ iff $X$ is precompact.

(ii) For every pair $(X, Y) \in \mathcal{B}_{E} \times \mathcal{B}_{E}$, we have

$$
X \subseteq Y \quad \Longrightarrow \quad \sigma(X) \leq \sigma(Y)
$$

(iii) For every $X \in \mathcal{B}_{E}$, we have

$$
\sigma(\bar{X})=\sigma(X)=\sigma(\operatorname{conv}(X))
$$

(iv) If $\left\{X_{n}\right\} \subseteq \mathcal{B}_{E}$ is a decreasing sequence (w.r.t. $\subseteq$ ) such that $\sigma\left(X_{n}\right) \rightarrow 0$ as $n \rightarrow \infty$, then the $X_{\infty}:=\bigcap_{n=1}^{\infty} \overline{X_{n}}$ is nonempty.

Observe that if $\sigma: \mathcal{B}_{E} \rightarrow[0, \infty)$ is a BG-measure of noncompactess on $E$, then $\sigma$ is a D-measure of noncompactess on $E$.

In this paper, using the axiomatic definition of the measure of noncompactness given by Dhage, we obtain new generalizations of Theorem 1.2. Finally, an existence result for a certain class of fractional integral equations will be given as an application.

\section{Main results}

Let $\mathcal{X}$ be a nonempty, bounded, closed, and convex subset of a Banach space $E$. We continue to use the same notations presented in the previous section of this paper.

Let $\mathcal{F}_{\mathcal{X}}^{\prime}$ be the set of self-mappings $D: \mathcal{X} \rightarrow \mathcal{X}$ satisfying the following conditions:

(i) $D$ is a continuous mapping.

(ii) There exists $\sigma: \mathcal{B}_{E} \rightarrow[0, \infty)$, a D-measure of noncompactness on $E$, such that for all $\varepsilon>0$, there exists some $\delta_{\varepsilon}>0$ for which

$$
W \in P(\mathcal{X}), \quad \varepsilon \leq \sigma(W)<\varepsilon+\delta_{\varepsilon} \quad \Longrightarrow \quad \sigma(D W)<\varepsilon .
$$

We have the following result.

Theorem 2.1 Let $D: \mathcal{X} \rightarrow \mathcal{X}$ be a mapping that belongs to $\mathcal{F}_{\mathcal{X}}^{\prime}$. Then $D$ has at least one fixed point.

The result of Theorem 2.1 can be obtained using the same arguments of the proof of Theorem 1.3 in [4]. By Theorem 2.1, we want just to mention that Theorem 1.3 is still valid for any D-measure of noncompactness.

Let $\mathcal{G}_{\mathcal{X}}$ be the set of mappings $D: \mathcal{X} \rightarrow \mathcal{X}$ such that

(i) $D$ is continuous.

(ii) There exists a function $\omega:[0, \infty) \rightarrow[0, \infty)$ such that

$\left(\omega_{1}\right) \omega(t)=0$ iff $t=0$;

$\left(\omega_{2}\right) \omega$ is nondecreasing and right continuous; 
$\left(\omega_{3}\right)$ for every $\varepsilon>0$, there exists $\gamma_{\varepsilon}>0$ such that

$$
W \in P(\mathcal{X}), \quad \varepsilon \leq \omega(\sigma(W))<\varepsilon+\gamma_{\varepsilon} \quad \Longrightarrow \quad \omega(\sigma(D W))<\varepsilon,
$$

where $\sigma: \mathcal{B}_{E} \rightarrow[0, \infty)$ is a $\mathrm{D}$-measure of noncompactness.

The following lemma can be proved using a similar argument as in the proof of Theorem 2.6 in [4].

\section{Lemma 2.2 We have}

$$
\mathcal{G}_{\mathcal{X}} \subseteq \mathcal{F}_{\mathcal{X}}^{\prime}
$$

Using Theorem 2.1 and Lemma 2.2, we obtain the following result.

Corollary 2.3 Let $D: \mathcal{X} \rightarrow \mathcal{X}$ be a mapping that belongs to $\mathcal{G}_{\mathcal{X}}$. Then $D$ has at least one fixed point.

Let $\Phi$ be the set of functions $\varphi:[0, \infty) \rightarrow[0, \infty)$ satisfying the conditions:

$\left(\Phi_{1}\right) \varphi \in L_{\text {loc }}^{1}[0, \infty)$;

$\left(\Phi_{2}\right)$ for every $\xi>0$, we have

$$
\int_{0}^{\xi} \varphi(s) d s>0
$$

Let $\mathcal{H}_{\mathcal{X}}$ be the set of mappings $D: \mathcal{X} \rightarrow \mathcal{X}$ such that

$\left(\mathcal{H}_{1}\right) D$ is continuous;

$\left(\mathcal{H}_{2}\right)$ for every $\varepsilon>0$, there exists some $\gamma_{\varepsilon}>0$ such that

$$
W \in P(\mathcal{X}), \quad \varepsilon \leq \int_{0}^{\sigma(W)} \varphi(s) d s<\varepsilon+\gamma_{\varepsilon} \Longrightarrow \int_{0}^{\sigma(D W)} \varphi(s) d s<\varepsilon,
$$

where $\varphi \in \Phi$ and $\sigma: \mathcal{B}_{E} \rightarrow[0, \infty)$ is a D-measure of noncompactness.

Lemma 2.4 We have

$$
\mathcal{H}_{\mathcal{X}} \subseteq \mathcal{G}_{\mathcal{X}}
$$

Proof Take

$$
\omega(t)=\int_{0}^{t} \varphi(s) d s, \quad t \geq 0,
$$

we obtain the desired result.

Using Corollary 2.3 and Lemma 2.4, we obtain the following result.

Corollary 2.5 Let $D: \mathcal{X} \rightarrow \mathcal{X}$ be a mapping that belongs to $\mathcal{H}_{\mathcal{X}}$. Then $D$ has at least one fixed point. 
Let $\mathcal{I}_{\mathcal{X}}$ be the set of mappings $D: \mathcal{X} \rightarrow \mathcal{X}$ such that

$\left(\mathcal{I}_{1}\right) D$ is continuous;

$\left(\mathcal{I}_{2}\right)$ there exists some $\varphi \in \Phi$ such that

$$
\int_{0}^{\sigma(D W)} \varphi(s) d s \leq k \int_{0}^{\sigma(W)} \varphi(s) d s, \quad W \in P(\mathcal{X})
$$

where $k \in(0,1)$ is a constant and $\sigma: \mathcal{B}_{E} \rightarrow[0, \infty)$ is a D-measure of noncompactness.

Lemma 2.6 We have

$$
\mathcal{I}_{\mathcal{X}} \subseteq \mathcal{H}_{\mathcal{X}}
$$

Proof Let $D: \mathcal{X} \rightarrow \mathcal{X}$ be a mapping that belongs to $\mathcal{I}_{\mathcal{X}}$. Let $\varepsilon>0$ be fixed. Let $\gamma_{\varepsilon}=\left(\frac{1}{k}-1\right) \varepsilon$. Take $W \in P(\mathcal{X})$ such that

$$
\varepsilon \leq \int_{0}^{\sigma(W)} \varphi(s) d s<\varepsilon+\gamma_{\varepsilon}=\frac{\varepsilon}{k} .
$$

From $\left(\mathcal{I}_{2}\right)$, we obtain

$$
\int_{0}^{\sigma(D W)} \varphi(s) d s \leq k \int_{0}^{\sigma(W)} \varphi(s) d s<k \frac{\varepsilon}{k}=\varepsilon
$$

so $D \in \mathcal{H}_{\mathcal{X}}$.

Using Corollary 2.5 and Lemma 2.6, we obtain the following result.

Corollary 2.7 Let $D: \mathcal{X} \rightarrow \mathcal{X}$ be a mapping that belongs to $\mathcal{I}_{\mathcal{X}}$. Then $D$ has at least one fixed point.

Remark 2.8 Take $\varphi(t)=1, t \geq 0$ in Corollary 2.7, we obtain Theorem 1.2.

Let $\mathcal{J}_{\mathcal{X}}$ be the set of mappings $D: \mathcal{X} \rightarrow \mathcal{X}$ such that

$\left(\mathcal{J}_{1}\right) D$ is continuous;

$\left(\mathcal{J}_{2}\right)$ there exists a function $\eta:(0, \infty) \rightarrow \mathbb{R}$ such that

$\left(\eta_{1}\right)$ for each sequence $\left\{\alpha_{n}\right\} \subset(0, \infty)$, we have

$$
\lim _{n \rightarrow \infty} \eta\left(\alpha_{n}\right)=-\infty \quad \Longrightarrow \quad \lim _{n \rightarrow \infty} \alpha_{n}=0
$$

$\left(\eta_{2}\right)$ there exists $\tau>0$ such that

$$
W \in P(\mathcal{X}), \quad \sigma(W) \sigma(D W)>0 \quad \Longrightarrow \quad \tau+\eta(\sigma(D W)) \leq \eta(\sigma(W))
$$

where $\sigma: \mathcal{B}_{E} \rightarrow[0, \infty)$ is a D-measure of noncompactness.

Theorem 2.9 Let $D: \mathcal{X} \rightarrow \mathcal{X}$ be a mapping that belongs to $\mathcal{J}_{\mathcal{X}}$. Then $D$ has at least one fixed point. 
Proof Consider the sequence $\left\{\mathcal{X}_{n}\right\}$ of subsets of $E$ defined by

$$
\left\{\begin{array}{l}
\mathcal{X}_{0}:=\mathcal{X}, \\
\mathcal{X}_{n+1}:=\overline{\operatorname{conv}}\left(D \mathcal{X}_{n}\right), \quad n=0,1,2, \ldots
\end{array}\right.
$$

By induction, we observe easily that

$$
\mathcal{X}_{n+1} \subseteq \mathcal{X}_{n}, \quad n=0,1,2, \ldots
$$

If for some $N$, we have $\sigma\left(\mathcal{X}_{N}\right)=0$, then by the property (i) of the D-measure of noncompactness, $\mathcal{X}_{N}$ is compact. Since $D\left(\mathcal{X}_{N}\right) \subseteq \mathcal{X}_{N}$ (from (2.2)), Schauder's fixed point theorem applied to the self-mapping $D: \mathcal{X}_{N} \rightarrow \mathcal{X}_{N}$ gives the desired result. So, without loss of the generality, we may assume that

$$
\sigma\left(\mathcal{X}_{n}\right)>0, \quad n=0,1,2, \ldots
$$

For $n=0$, since $\sigma\left(\mathcal{X}_{0}\right)>0$ and $\sigma\left(D \mathcal{X}_{0}\right)=\sigma\left(\mathcal{X}_{1}\right)>0$, from the property $\left(\eta_{2}\right)$ we have

$$
\tau+\eta\left(\sigma\left(D \mathcal{X}_{0}\right)\right) \leq \eta\left(\sigma\left(\mathcal{X}_{0}\right)\right)
$$

which yields

$$
\eta\left(\sigma\left(\mathcal{X}_{1}\right)\right) \leq \eta\left(\sigma\left(\mathcal{X}_{0}\right)\right)-\tau
$$

Similarly, for $n=1$, we have

$$
\eta\left(\sigma\left(\mathcal{X}_{2}\right)\right) \leq \eta\left(\sigma\left(\mathcal{X}_{1}\right)\right)-\tau \leq \eta\left(\sigma\left(\mathcal{X}_{0}\right)\right)-2 \tau \text {. }
$$

By induction, we obtain

$$
\eta\left(\sigma\left(\mathcal{X}_{n}\right)\right) \leq \eta\left(\sigma\left(\mathcal{X}_{0}\right)\right)-n \tau, \quad n=0,1,2, \ldots
$$

Since

$$
\lim _{n \rightarrow \infty} \eta\left(\sigma\left(\mathcal{X}_{0}\right)\right)-n \tau=-\infty
$$

we deduce that

$$
\lim _{n \rightarrow \infty} \eta\left(\sigma\left(\mathcal{X}_{n}\right)\right)=-\infty
$$

so from the property $\left(\eta_{1}\right)$ we have

$$
\lim _{n \rightarrow \infty} \sigma\left(\mathcal{X}_{n}\right)=0
$$

From the property (iv) of the D-measure of noncompactness, the set $M:=\bigcap_{n=1}^{\infty} \mathcal{X}_{n}$ is nonempty. Moreover, for every $p=0,1,2, \ldots$, we have

$$
M \subseteq \mathcal{X}_{p}
$$


which implies from (2.2) that

$$
D M \subseteq D \mathcal{X}_{p} \subseteq \mathcal{X}_{p+1} \subseteq \mathcal{X}_{p}, \quad p=0,1,2, \ldots
$$

Then $D: M \rightarrow M$ is well defined. On the other hand, from (2.4) and the property (ii) of the D-measure of noncompactness, we have

$$
\sigma(M) \leq \sigma\left(\mathcal{X}_{p}\right), \quad p=0,1,2, \ldots
$$

Passing to the limit as $p \rightarrow \infty$ and using (2.3), we obtain

$$
\sigma(M)=0,
$$

which implies from the property (i) of the D-measure of noncompactness that $\bar{M}=M$ is compact. Applying Schauder's fixed point theorem to the mapping $D: M \rightarrow M$, we obtain the desired result.

Remark 2.10 Observe that $\mathcal{D}_{\mathcal{X}} \subseteq \mathcal{J}_{\mathcal{X}}$. In fact, if $D: \mathcal{X} \rightarrow \mathcal{X}$ belongs to $\mathcal{D}_{\mathcal{X}}$, that is,

$$
\sigma(D W) \leq k \sigma(W), \quad W \in P(\mathcal{X})
$$

then

$$
W \in P(X), \quad \sigma(W) \sigma(D W)>0 \quad \Longrightarrow \quad \ln \sigma(D W)-\ln k \leq \ln \sigma(W) .
$$

Then $D \in \mathcal{J}_{\mathcal{X}}$ with $\eta(t)=\ln t, t>0$. Therefore, Theorem 2.9 is a generalization of Theorem 1.2.

Let $\mathcal{K}_{\mathcal{X}}$ be the set of mappings $D: \mathcal{X} \rightarrow \mathcal{X}$ such that

$\left(\mathcal{K}_{1}\right) D$ is continuous;

$\left(\mathcal{K}_{2}\right)$ there exists a function $\theta:(0, \infty) \rightarrow(1, \infty)$ such that

$\left(\theta_{1}\right)$ for each sequence $\left\{u_{n}\right\} \subset(0, \infty)$, we have

$$
\lim _{n \rightarrow \infty} \theta\left(u_{n}\right)=1 \quad \Longrightarrow \quad \lim _{n \rightarrow \infty} u_{n}=0
$$

$\left(\theta_{2}\right)$ there exist $k \in(0,1)$ and a D-measure of noncompactness $\sigma: \mathcal{B}_{E} \rightarrow[0, \infty)$ such that

$$
W \in P(\mathcal{X}), \quad \sigma(W) \sigma(D W)>0 \quad \Longrightarrow \quad \theta(\sigma(D W)) \leq[\theta(\sigma(W))]^{k}
$$

We have the following result.

Theorem 2.11 Let $D: \mathcal{X} \rightarrow \mathcal{X}$ be a mapping that belongs to $\mathcal{K}_{\mathcal{X}}$. Then $D$ has at least one fixed point. 
Proof Consider the sequence $\left\{\mathcal{X}_{n}\right\}$ of subsets of $E$ defined by (2.1). As in the proof of Theorem 2.9, without loss of the generality, we may assume that

$$
\sigma\left(\mathcal{X}_{n}\right)>0, \quad n=0,1,2, \ldots
$$

For $n=0$, since $\sigma\left(\mathcal{X}_{0}\right)>0$ and $\sigma\left(D \mathcal{X}_{0}\right)=\sigma\left(\mathcal{X}_{1}\right)>0$, we have

$$
\theta\left(\sigma\left(D \mathcal{X}_{0}\right)\right) \leq\left[\theta\left(\sigma\left(\mathcal{X}_{0}\right)\right)\right]^{k}
$$

that is,

$$
\theta\left(\sigma\left(\mathcal{X}_{1}\right)\right) \leq\left[\theta\left(\sigma\left(\mathcal{X}_{0}\right)\right)\right]^{k}
$$

Again, for $n=1$, since $\sigma\left(\mathcal{X}_{1}\right)>0$ and $\sigma\left(D \mathcal{X}_{1}\right)=\sigma\left(\mathcal{X}_{2}\right)>0$, we have

$$
\theta\left(\sigma\left(D \mathcal{X}_{1}\right)\right) \leq\left[\theta\left(\sigma\left(\mathcal{X}_{1}\right)\right)\right]^{k}
$$

that is,

$$
\theta\left(\sigma\left(\mathcal{X}_{2}\right)\right) \leq\left[\theta\left(\sigma\left(\mathcal{X}_{1}\right)\right)\right]^{k}
$$

So

$$
\theta\left(\sigma\left(\mathcal{X}_{2}\right)\right) \leq\left[\theta\left(\sigma\left(\mathcal{X}_{0}\right)\right)\right]^{k^{2}}
$$

Therefore, by induction, we get

$$
1<\theta\left(\sigma\left(\mathcal{X}_{n}\right)\right) \leq\left[\theta\left(\sigma\left(\mathcal{X}_{0}\right)\right)\right]^{k^{n}}, \quad n=0,1,2, \ldots
$$

Passing to the limit as $n \rightarrow \infty$, we obtain

$$
\lim _{n \rightarrow \infty} \theta\left(\sigma\left(\mathcal{X}_{n}\right)\right)=1
$$

so from the property $\left(\theta_{1}\right)$ we have

$$
\lim _{n \rightarrow \infty} \sigma\left(\mathcal{X}_{n}\right)=0
$$

The rest of the proof is similar to that in the proof of Theorem 2.9.

Corollary 2.12 Let $D: \mathcal{X} \rightarrow \mathcal{X}$ be a continuous mapping. Suppose that there exist a constant $k \in(0,1)$ and a D-measure of noncompactness $\sigma: \mathcal{B}_{E} \rightarrow[0, \infty)$ such that

$$
2-\frac{2}{\pi} \arctan \left(\frac{1}{\sqrt{\sigma(D W)}}\right) \leq\left[2-\frac{2}{\pi} \arctan \left(\frac{1}{\sqrt{\sigma(W)}}\right)\right]^{k}
$$

for any $W \in P(\mathcal{X})$ with $\sigma(W) \sigma(D W)>0$. Then $D$ has at least one fixed point. 
Proof Taking

$$
\theta(t)=2-\frac{2}{\pi} \arctan \left(\frac{1}{\sqrt{t}}\right), \quad t>0,
$$

in Theorem 2.11, we obtain the desired result.

Remark 2.13 Observe that $\mathcal{D}_{\mathcal{X}} \subseteq \mathcal{K}_{\mathcal{X}}$. In fact, if $D: \mathcal{X} \rightarrow \mathcal{X}$ belongs to $\mathcal{D}_{\mathcal{X}}$, that is,

$$
\sigma(D W) \leq k \sigma(W), \quad W \in P(\mathcal{X})
$$

then

$$
W \in P(\mathcal{X}), \quad \sigma(W) \sigma(D W)>0 \quad \Longrightarrow \quad e^{\sigma(D W)} \leq\left[e^{\sigma(W)}\right]^{k}
$$

Therefore $D \in \mathcal{K}_{\mathcal{X}}$ with $\theta(t)=e^{t}$.

Let $\mathcal{L}_{\mathcal{X}}$ be the set of mappings $D: \mathcal{X} \rightarrow \mathcal{X}$ such that

$\left(\mathcal{L}_{1}\right) \quad D$ is continuous;

$\left(\mathcal{L}_{2}\right)$ there exists a function $\zeta:[0, \infty) \times[0, \infty) \rightarrow \mathbb{R}$ such that

$\left(\zeta_{1}\right) \zeta\left(z_{1}, z_{2}\right)<z_{2}-z_{1}$, for all $z_{1}, z_{2}>0$;

$\left(\zeta_{2}\right)$ if $\left\{u_{n}\right\}$ and $\left\{v_{n}\right\}$ are two sequences in $(0, \infty)$ such that $\lim _{n \rightarrow \infty} u_{n}=\lim _{n \rightarrow \infty} v_{n}=$ $\ell>0$, then

$$
\limsup _{n \rightarrow \infty} \zeta\left(u_{n}, v_{n}\right)<0
$$

$\left(\zeta_{3}\right)$

$$
\zeta(\sigma(D W), \sigma(W)) \geq 0, \quad W \in P(\mathcal{X})
$$

where $\sigma: \mathcal{B}_{E} \rightarrow[0, \infty)$ is a D-measure of noncompactness.

Theorem 2.14 Let $D: \mathcal{X} \rightarrow \mathcal{X}$ be a mapping that belongs to $\mathcal{L}_{\mathcal{X}}$. Then $D$ has at least one fixed point.

Proof Consider the sequence $\left\{\mathcal{X}_{n}\right\}$ of subsets of $E$ defined by (2.1). From the property $\left(\zeta_{3}\right)$, we have

$$
\zeta\left(\sigma\left(\mathcal{X}_{n+1}\right), \sigma\left(\mathcal{X}_{n}\right)\right) \geq 0, \quad n=0,1,2, \ldots
$$

As before, without loss of the generality, we may assume that

$$
\sigma\left(\mathcal{X}_{n}\right)>0, \quad n=0,1,2, \ldots
$$

From the property $\left(\zeta_{1}\right),(2.5)$ and (2.6), we get

$$
\sigma\left(\mathcal{X}_{n}\right) \geq \sigma\left(\mathcal{X}_{n+1}\right), \quad n=0,1,2, \ldots
$$


Then there is some $r \geq 0$ such that

$$
\lim _{n \rightarrow \infty} \sigma\left(\mathcal{X}_{n}\right)=r
$$

If $r>0$, then from the property $\left(\zeta_{2}\right)$, we have

$$
\limsup _{n \rightarrow \infty} \zeta\left(\sigma\left(\mathcal{X}_{n+1}\right), \sigma\left(\mathcal{X}_{n}\right)\right)<0
$$

which contradicts (2.5). As consequence, we have

$$
\lim _{n \rightarrow \infty} \sigma\left(\mathcal{X}_{n}\right)=0
$$

The rest of the proof is similar to the proof of Theorem 2.9.

Remark 2.15 Taking

$$
\zeta\left(z_{1}, z_{2}\right)=k z_{2}-z_{1}
$$

where $k \in(0,1)$ is a constant, we obtain Theorem 1.2.

Corollary 2.16 Let $D: \mathcal{X} \rightarrow \mathcal{X}$ be a continuous mapping such that

$$
\sigma(D W) \leq \sigma(W)-\Phi(\sigma(W)), \quad W \in P(\mathcal{X})
$$

where $\Phi:[0, \infty) \rightarrow[0, \infty)$ is a lower semi-continuous function with $\Phi^{-1}(0)=\{0\}$ and $\sigma:$ $\mathcal{B}_{E} \rightarrow[0, \infty)$ is a D-measure of noncompactness. Then D has at least one fixed point.

Proof Taking

$$
\zeta\left(z_{1}, z_{2}\right)=z_{2}-\Phi\left(z_{2}\right)-z_{1}
$$

in Theorem 2.14, we obtain the desired result.

Corollary 2.17 Let $D: \mathcal{X} \rightarrow \mathcal{X}$ be a continuous mapping such that

$$
\sigma(D W) \leq \psi(\sigma(W)), \quad W \in P(\mathcal{X})
$$

where $\psi:[0, \infty) \rightarrow[0, \infty)$ is an upper semi-continuous function with $\psi(t)<t$ for all $t>0$ and $\sigma: \mathcal{B}_{E} \rightarrow[0, \infty)$ is a D-measure of noncompactness. Then $D$ has at least one fixed point.

Proof Taking

$$
\zeta\left(z_{1}, z_{2}\right)=\psi\left(z_{2}\right)-z_{1}
$$

in Theorem 2.14, we obtain the desired result. 


\section{An existence result for a fractional integral equation}

The measure of noncompactness argument is a useful tool in Nonlinear Analysis. In particular, such argument can be used to obtain existence results for various classes of integral equations. For more details on the applications of the measure of noncompactness concept, we refer the reader to $[1,3,4,6,8-15]$ and the references therein.

In this section, we discuss the existence of solutions to the fractional integral equation

$$
y(t)=\frac{f(t, y(t))}{\Gamma(\alpha)} \int_{0}^{t} \frac{g^{\prime}(s) u(s, y(s))}{(g(t)-g(s))^{1-\alpha}} d s, \quad t \in[0, T]
$$

where $T>0, \alpha \in(0,1), u, f:[0, T] \times \mathbb{R} \rightarrow \mathbb{R}$ and $g:[0, T] \rightarrow \mathbb{R}$.

We suppose that the following conditions are satisfied.

(i) The function $f:[0, T] \times \mathbb{R} \rightarrow \mathbb{R}$ is continuous.

(ii) There exists an upper semi-continuous function $\psi:[0, \infty) \rightarrow[0, \infty)$ such that $\psi(0)=0, \psi(t)<t$ for all $t>0, \psi$ is nondecreasing, and

$$
|f(t, x)-f(t, y)| \leq \psi(|x-y|), \quad(t, x, y) \in[0, T] \times \mathbb{R} \times \mathbb{R}
$$

(iii) The function $u:[0, \infty) \rightarrow[0, \infty)$ is continuous and there exists a nondecreasing function $\omega:[0, \infty) \rightarrow[0, \infty)$ such that

$$
|u(t, z)| \leq \omega(|z|), \quad(t, z) \in[0, T] \times \mathbb{R} .
$$

(iv) The function $g:[0, T] \rightarrow \mathbb{R}$ is $C^{1}$ and nondecreasing.

(v) There exists $r_{0}>0$ such that

$$
\left(\psi\left(r_{0}\right)+F\right) \omega\left(r_{0}\right)(g(T)-g(0))^{\alpha} \leq r_{0} \Gamma(\alpha+1)
$$

and

$$
\frac{\omega\left(r_{0}\right)}{\Gamma(\alpha+1)}(g(T)-g(0))^{\alpha} \leq 1,
$$

where $F=\max \{\mid f(t, 0): t \in[0, T]\}$.

Let $E=C([0, T] ; \mathbb{R})$ be the set of real continuous functions defined in $[0, T]$. The set $E$ endowed with the norm

$$
\|z\|=\max \{|z(t)|: t \in[0, T]\}, \quad z \in E,
$$

is a Banach space. Let $W$ be a nonempty and bounded subset of $E$. Let us define the mapping $\gamma: W \times[0, \infty) \rightarrow[0, \infty)$ by

$$
\gamma(z, \rho)=\sup \{|z(a)-z(b)|: a, b \in[0, T],|a-b| \leq \rho\}, \quad z \in W, \rho \geq 0 .
$$

Set

$$
\gamma(W, \rho)=\sup \{\gamma(z, \rho): z \in W\}, \quad \rho \geq 0 .
$$


Let $\mathcal{B}_{E}$ be the set of all nonempty bounded subsets of $E$. Then the mapping

$$
\sigma: \mathcal{B}_{E} \rightarrow[0, \infty)
$$

defined by

$$
\sigma(W)=\lim _{\rho \rightarrow 0^{+}} \gamma(W, \rho), \quad W \in \mathcal{B}_{E}
$$

is a BG-measure of noncompactness (then it is a D-measure of noncompactness) on the space $E$ (see [1]).

We have the following existence result.

Theorem 3.1 Under the assumptions (i)-(v), equation (3.1) has at least one solution $y^{*} \in E$. Moreover, we have $\left\|y^{*}\right\| \leq r_{0}$.

Proof Let us consider the operator $D$ defined on $E$ by

$$
(D y)(t)=\frac{f(t, y(t))}{\Gamma(\alpha)} \int_{0}^{t} \frac{g^{\prime}(s) u(s, y(s))}{(g(t)-g(s))^{1-\alpha}} d s, \quad(y, t) \in E \times[0, T] .
$$

At first, we show that the operator $D$ maps $E$ into itself. Set

$$
(H y)(t)=\int_{0}^{t} \frac{g^{\prime}(s) u(s, y(s))}{(g(t)-g(s))^{1-\alpha}} d s, \quad(y, t) \in E \times[0, T] .
$$

From the assumption (i), we have just to show that $H$ maps $E$ into itself. In order to prove this fact, let us fix some $y \in E$. Observe that $H y:[0, T] \rightarrow \mathbb{R}$ is a well-defined function. In fact, using the assumptions (iii) and (iv), for all $t \in[0, T]$ we have

$$
\begin{aligned}
|(H y)(t)| & \leq \int_{0}^{t} \frac{g^{\prime}(s)|u(s, y(s))|}{(g(t)-g(s))^{1-\alpha}} d s \\
& \leq \omega(\|y\|) \int_{0}^{t} \frac{g^{\prime}(s)}{(g(t)-g(s))^{1-\alpha}} d s \\
& =\frac{\omega(\|y\|)}{\alpha}(g(t)-g(0))^{\alpha},
\end{aligned}
$$

that is,

$$
|(H y)(t)| \leq \frac{\omega(\|y\|)}{\alpha}(g(t)-g(0))^{\alpha}<\infty, \quad t \in[0, T] .
$$

Let us prove the continuity of $H y$ at 0 . To do this, let $\left\{t_{n}\right\}$ be a sequence in $[0, T]$ such that $t_{n} \rightarrow 0^{+}$as $n \rightarrow \infty$. From (3.4), for all $n$ we have

$$
\left|(H y)\left(t_{n}\right)\right| \leq \frac{\omega(\|y\|)}{\alpha}\left(g\left(t_{n}\right)-g(0)\right)^{\alpha}
$$

Passing to the limit as $n \rightarrow \infty$ and using the continuity of $g$ at 0 , we obtain

$$
\lim _{n \rightarrow \infty}(H y)\left(t_{n}\right)=0=(H y)(0) .
$$

Then $H y$ is continuous at 0 . 
Now, let $t \in(0, T]$ be fixed and $\left\{t_{n}\right\}$ be a sequence in $(0, T]$ such that $t_{n} \rightarrow t$ as $n \rightarrow \infty$. Without restriction of the generality, we may assume that $t_{n} \geq t$ for $n$ large enough. For every $n$, we have

$$
\left|(H y)\left(t_{n}\right)-(H y)(t)\right|=\left|\int_{0}^{t_{n}} \frac{g^{\prime}(s) u(s, y(s))}{\left(g\left(t_{n}\right)-g(s)\right)^{1-\alpha}} d s-\int_{0}^{t} \frac{g^{\prime}(s) u(s, y(s))}{(g(t)-g(s))^{1-\alpha}} d s\right| .
$$

For $n$ large enough, we can write

$$
\begin{aligned}
\left|(H y)\left(t_{n}\right)-(H y)(t)\right| \leq & \left|\int_{0}^{t}\left(\frac{g^{\prime}(s) u(s, y(s))}{\left(g\left(t_{n}\right)-g(s)\right)^{1-\alpha}}-\frac{g^{\prime}(s) u(s, y(s))}{(g(t)-g(s))^{1-\alpha}}\right) d \tau\right| \\
& +\left|\int_{t}^{t_{n}} \frac{g^{\prime}(s) u(s, y(s))}{\left(g\left(t_{n}\right)-g(s)\right)^{1-\alpha}} d s\right| \\
\leq & \omega(\|y\|) \int_{0}^{t}\left(\frac{g^{\prime}(s)}{(g(t)-g(s))^{1-\alpha}}-\frac{g^{\prime}(s)}{\left(g\left(t_{n}\right)-g(s)\right)^{1-\alpha}}\right) d s \\
& +\omega(\|y\|) \int_{t}^{t_{n}} \frac{g^{\prime}(s)}{\left(g\left(t_{n}\right)-g(s)\right)^{1-\alpha}} d s \\
= & \frac{\omega(\|y\|)}{\alpha}\left((g(t)-g(0))^{\alpha}+\left(g\left(t_{n}\right)-g(t)\right)^{\alpha}-\left(g\left(t_{n}\right)-g(0)\right)^{\alpha}\right) \\
& +\frac{\omega(\|y\|)}{\alpha}\left(g\left(t_{n}\right)-g(t)\right)^{\alpha} .
\end{aligned}
$$

Since $g$ is continuous in $[0, T]$, we have

$$
\lim _{n \rightarrow \infty} \frac{\omega(\|y\|)}{\alpha}\left((g(t)-g(0))^{\alpha}+\left(g\left(t_{n}\right)-g(t)\right)^{\alpha}-\left(g\left(t_{n}\right)-g(0)\right)^{\alpha}\right)+\frac{\omega(\|y\|)}{\alpha}\left(g\left(t_{n}\right)-g(t)\right)^{\alpha}=0,
$$

which yields $\lim _{n \rightarrow \infty}\left|(H y)\left(t_{n}\right)-(H y)(t)\right|=0$. Then $H y$ is continuous at $t$. As consequence, $H y \in E$, for all $y \in E$, and $D: E \rightarrow E$ is well defined.

On the other hand, using the assumptions (ii) and (iii), for an arbitrarily fixed $y \in E$ and $t \in[0, T]$, we have

$$
\begin{aligned}
|(D y)(t)| & \leq \frac{|f(t, y(t))|}{\Gamma(\alpha)} \int_{0}^{t} \frac{g^{\prime}(s)|u(s, y(s))|}{(g(t)-g(s))^{1-\alpha}} d s \\
& \leq \frac{|f(t, y(t))-f(t, 0)|+|f(t, 0)|}{\Gamma(\alpha)} \int_{0}^{t} \frac{g^{\prime}(s) \omega(|y(s)|)}{(g(t)-g(s))^{1-\alpha}} d s \\
& \leq \frac{(\psi(|y(t)|)+F) \omega(\|y\|)}{\Gamma(\alpha+1)}(g(t)-g(0))^{\alpha} \\
& \leq \frac{(\psi(\|y\|)+F) \omega(\|y\|)}{\Gamma(\alpha+1)}(g(T)-g(0))^{\alpha} .
\end{aligned}
$$

Then

$$
\|D y\| \leq \frac{(\psi(\|y\|)+F) \omega(\|y\|)}{\Gamma(\alpha+1)}(g(T)-g(0))^{\alpha}, \quad y \in E .
$$

Using the above inequality, the fact that the functions $\psi, \omega:[0, \infty) \rightarrow[0, \infty)$ are nondecreasing, and the assumption (v), we infer that the operator $D$ maps $\overline{B\left(0, r_{0}\right)}$ into itself, 
where

$$
\overline{B\left(0, r_{0}\right)}=\left\{z \in E:\|z\| \leq r_{0}\right\}
$$

Now, we claim that the operator $D: \overline{B\left(0, r_{0}\right)} \rightarrow \overline{B\left(0, r_{0}\right)}$ is continuous. From (3.2), we can write $D$ in the form

$$
D y=\frac{1}{\Gamma(\alpha)} G y \cdot H y, \quad y \in E,
$$

where

$$
(G y)(t)=f(t, y(t)), \quad(y, t) \in E \times[0, T],
$$

and $H y$ is defined by (3.3). In order to prove our claim, it is sufficient to show that the operators $G$ and $H$ are continuous on $\overline{B\left(0, r_{0}\right)}$. First of all, we show that $G$ is a continuous operator on $\overline{B\left(0, r_{0}\right)}$. To do this, we take a sequence $\left\{y_{n}\right\} \subset \overline{B\left(0, r_{0}\right)}$ and $y \in \overline{B\left(0, r_{0}\right)}$ such that $\left\|y_{n}-y\right\| \rightarrow 0$ as $n \rightarrow \infty$, and we have to prove that $\left\|G y_{n}-G y\right\| \rightarrow 0$ as $n \rightarrow \infty$. In fact, for all $t \in[0, T]$, using the condition (ii), we have

$$
\begin{aligned}
\left|\left(G y_{n}\right)(t)-(G y)(t)\right| & =\left|f\left(t, y_{n}(t)\right)-f(t, y(t))\right| \\
& \leq \psi\left(\left|y_{n}(t)-y(t)\right|\right) \\
& \leq \psi\left(\left\|y_{n}-y\right\|\right) \\
& \leq\left\|y_{n}-y\right\| .
\end{aligned}
$$

Thus we have

$$
\left\|G y_{n}-G y\right\| \leq\left\|y_{n}-y\right\|, \quad \text { for all } n \text {. }
$$

Passing to the limit as $n \rightarrow \infty$ in the above inequality, we obtain

$$
\lim _{n \rightarrow \infty}\left\|G y_{n}-G y\right\|=0
$$

This proves that $G$ is a continuous operator on $\overline{B\left(0, r_{0}\right)}$. Next, we show that $H$ is a continuous operator on $\overline{B\left(0, r_{0}\right)}$. To do this, we fix a real number $\varepsilon>0$ and we take arbitrary functions $x, y \in \overline{B\left(0, r_{0}\right)}$ such that $\|x-y\|<\varepsilon$. For all $t \in[0, T]$, we have

$$
\begin{aligned}
|(H x)(t)-(H y)(t)| & \leq \int_{0}^{t} \frac{g^{\prime}(s)|u(s, x(s))-u(s, y(s))|}{(g(t)-g(s))^{1-\alpha}} d s \\
& \leq u\left(r_{0}, \varepsilon\right) \int_{0}^{t} \frac{g^{\prime}(s)}{(g(t)-g(s))^{1-\alpha}} d s \\
& \leq \frac{u\left(r_{0}, \varepsilon\right)}{\alpha}(g(T)-g(0))^{\alpha},
\end{aligned}
$$

where

$$
u\left(r_{0}, \varepsilon\right)=\sup \left\{|u(\tau, v)-u(\tau, w)|: \tau \in[0, T], v, w \in\left[-r_{0}, r_{0}\right],|v-w|<\varepsilon\right\} .
$$


Therefore,

$$
\|H x-H y\| \leq \frac{u\left(r_{0}, \varepsilon\right)}{\alpha}(g(T)-g(0))^{\alpha} .
$$

Since $u$ is uniformly continuous on the compact $[0, T] \times\left[-r_{0}, r_{0}\right]$, we have $u\left(r_{0}, \varepsilon\right) \rightarrow 0$ as $\varepsilon \rightarrow 0^{+}$and, therefore, the last inequality gives us

$$
\lim _{\varepsilon \rightarrow 0^{+}}\|H x-H y\|=0
$$

Then $H$ is continuous on $\overline{B\left(0, r_{0}\right)}$ and $D$ maps continuously the set $\overline{B\left(0, r_{0}\right)}$ into itself.

Further, let $W$ be a nonempty subset of $\overline{B\left(0, r_{0}\right)}$. Let $\rho>0$ be fixed, $y \in W$, and $t_{1}, t_{2} \in$ $[0, T]$ be such that $\left|t_{1}-t_{2}\right| \leq \rho$. Without restriction of the generality, we may assume that $t_{1} \geq t_{2}$. We have

$$
\begin{aligned}
& \left|(D y)\left(t_{1}\right)-(D y)\left(t_{2}\right)\right| \\
& \leq\left|\frac{f\left(t_{1}, y\left(t_{1}\right)\right)}{\Gamma(\alpha)} \int_{0}^{t_{1}} \frac{g^{\prime}(s) u(s, y(s))}{\left(g\left(t_{1}\right)-g(s)\right)^{1-\alpha}} d s-\frac{f\left(t_{2}, y\left(t_{2}\right)\right)}{\Gamma(\alpha)} \int_{0}^{t_{2}} \frac{g^{\prime}(s) u(s, y(s))}{\left(g\left(t_{2}\right)-g(s)\right)^{1-\alpha}} d s\right| \\
& \leq\left|\frac{f\left(t_{1}, y\left(t_{1}\right)\right)}{\Gamma(\alpha)} \int_{0}^{t_{1}} \frac{g^{\prime}(s) u(s, y(s))}{\left(g\left(t_{1}\right)-g(s)\right)^{1-\alpha}} d s-\frac{f\left(t_{2}, y\left(t_{2}\right)\right)}{\Gamma(\alpha)} \int_{0}^{t_{1}} \frac{g^{\prime}(s) u(s, y(s))}{\left(g\left(t_{1}\right)-g(s)\right)^{1-\alpha}} d s\right| \\
& +\frac{\left|f\left(t_{2}, y\left(t_{2}\right)\right)\right|}{\Gamma(\alpha)}\left|\int_{0}^{t_{1}} \frac{g^{\prime}(s) u(s, y(s))}{\left(g\left(t_{1}\right)-g(s)\right)^{1-\alpha}} d s-\int_{0}^{t_{2}} \frac{g^{\prime}(s) u(s, y(s))}{\left(g\left(t_{2}\right)-g(s)\right)^{1-\alpha}} d s\right| \\
& \leq \frac{\left|f\left(t_{1}, y\left(t_{1}\right)\right)-f\left(t_{1}, y\left(t_{2}\right)\right)\right|+\left|f\left(t_{1}, y\left(t_{2}\right)\right)-f\left(t_{2}, y\left(t_{2}\right)\right)\right|}{\Gamma(\alpha)} \int_{0}^{t_{1}} \frac{g^{\prime}(s)|u(s, y(s))|}{\left(g\left(t_{1}\right)-g(s)\right)^{1-\alpha}} d s \\
& +\frac{\left|f\left(t_{2}, y\left(t_{2}\right)\right)-f\left(t_{2}, 0\right)\right|+\left|f\left(t_{2}, 0\right)\right|}{\Gamma(\alpha)} \mid \int_{0}^{t_{1}} \frac{g^{\prime}(s) u(s, y(s))}{\left(g\left(t_{1}\right)-g(s)\right)^{1-\alpha}} d s \\
& -\int_{0}^{t_{2}} \frac{g^{\prime}(s) u(s, y(s))}{\left(g\left(t_{1}\right)-g(s)\right)^{1-\alpha}} d s \\
& +\frac{\left|f\left(t_{2}, y\left(t_{2}\right)\right)-f\left(t_{2}, 0\right)\right|+\left|f\left(t_{2}, 0\right)\right|}{\Gamma(\alpha)} \mid \int_{0}^{t_{2}} \frac{g^{\prime}(s) u(s, y(s))}{\left(g\left(t_{1}\right)-g(s)\right)^{1-\alpha}} d s \\
& -\int_{0}^{t_{2}} \frac{g^{\prime}(s) u(s, y(s))}{\left(g\left(t_{2}\right)-g(s)\right)^{1-\alpha}} d s \\
& \leq \frac{\psi\left(\left|y\left(t_{1}\right)-y\left(t_{2}\right)\right|\right)+\omega_{f}\left(r_{0}, \rho\right)}{\Gamma(\alpha+1)} \omega\left(r_{0}\right)\left(g\left(t_{1}\right)-g(0)\right)^{\alpha} \\
& +\frac{\left(\psi\left(r_{0}\right)+F\right) \omega\left(r_{0}\right)}{\Gamma(\alpha)} \int_{t_{2}}^{t_{1}} \frac{g^{\prime}(s)}{\left(g\left(t_{1}\right)-g(s)\right)^{1-\alpha}} d s \\
& +\frac{\left(\psi\left(r_{0}\right)+F\right) \omega\left(r_{0}\right)}{\Gamma(\alpha+1)}\left(\left(g\left(t_{2}\right)-g(0)\right)^{\alpha}+\left(g\left(t_{1}\right)-g\left(t_{2}\right)\right)^{\alpha}-\left(g\left(t_{1}\right)-g(0)\right)^{\alpha}\right) \\
& \leq \frac{\psi(\gamma(y, \rho))+\omega_{f}\left(r_{0}, \rho\right)}{\Gamma(\alpha+1)} \omega\left(r_{0}\right)(g(T)-g(0))^{\alpha}+\frac{2\left(\psi\left(r_{0}\right)+F\right) \omega\left(r_{0}\right)}{\Gamma(\alpha+1)}\left(g\left(t_{1}\right)-g\left(t_{2}\right)\right)^{\alpha} \\
& \leq \frac{\psi(\gamma(\gamma, \rho))+\omega_{f}\left(r_{0}, \rho\right)}{\Gamma(\alpha+1)} \omega\left(r_{0}\right)(g(T)-g(0))^{\alpha}+\frac{2\left(\psi\left(r_{0}\right)+F\right) \omega\left(r_{0}\right)}{\Gamma(\alpha+1)}[\gamma(g, \rho)]^{\alpha},
\end{aligned}
$$


where

$$
\omega_{f}\left(r_{0}, \rho\right)=\sup \left\{|f(t, u)-f(s, u)|: u \in\left[-r_{0}, r_{0}\right], t, s \in[0, T],|t-s| \leq \rho\right\} .
$$

Therefore,

$$
\gamma(D W, \rho) \leq \frac{\psi(\gamma(W, \rho))+\omega_{f}\left(r_{0}, \rho\right)}{\Gamma(\alpha+1)} \omega\left(r_{0}\right)(g(T)-g(0))^{\alpha}+\frac{2\left(\psi\left(r_{0}\right)+F\right) \omega\left(r_{0}\right)}{\Gamma(\alpha+1)}[\omega(g, \rho)]^{\alpha} .
$$

Passing to the limit superior as $\rho \rightarrow 0^{+}$and using the fact that $\psi$ is upper semi-continuous, we obtain

$$
\sigma(D W) \leq \frac{\psi(\sigma(W))}{\Gamma(\alpha+1)} \omega\left(r_{0}\right)(g(T)-g(0))^{\alpha} .
$$

Then, from the assumption (v), we obtain

$$
\sigma(D W) \leq \psi(\sigma(W))
$$

As a consequence, for any nonempty subsets $W$ of $\overline{B\left(0, r_{0}\right)}$, we have

$$
\zeta(\sigma(D W), \sigma(W)) \geq 0
$$

where $\zeta:[0, \infty) \times[0, \infty) \rightarrow \mathbb{R}$ is defined by

$$
\zeta\left(z_{1}, z_{2}\right)=\psi\left(z_{2}\right)-z_{1}, \quad\left(z_{1}, z_{2}\right) \in[0, \infty) \times[0, \infty)
$$

Under the assumptions on the function $\psi$, the operator $D: \overline{B\left(0, r_{0}\right)} \rightarrow \overline{B\left(0, r_{0}\right)}$ belongs to the family of operators $\mathcal{L}_{\mathcal{X}}$, where $\mathcal{X}=\overline{B\left(0, r_{0}\right)}$. Then by Theorem 2.14 , we deduce that $D$ has at least one fixed point $y^{*} \in \overline{B\left(0, r_{0}\right)}$, which is a solution to equation (3.1).

\section{Competing interests}

The authors declare that they have no competing interests.

\section{Authors' contributions}

All authors contributed equally to the writing of this paper. All authors read and approved the final manuscript.

\section{Author details}

'Department of Mathematics, College of Science, King Saud University, P.O. Box 2455, Riyadh, 11451, Saudi Arabia. ${ }^{2}$ Department of Mathematics, Atilim University, Incek, Ankara, 06836, Turkey. ${ }^{3}$ Nonlinear Analysis and Applied Mathematics Research Group (NAAM), King Abdulaziz University, Jeddah, 21589, Saudi Arabia. ${ }^{4}$ School of Mathematics, Statistics and Applied Mathematics, National University of Ireland, Galway, Ireland.

\section{Acknowledgements}

The first and fourth authors would like to extend their sincere appreciations to the Deanship of Scientific Research at King Saud University for its funding of this Prolific Research Group Project No. PRG-1436-10.

Received: 17 September 2015 Accepted: 4 January 2016 Published online: 13 January 2016

\section{References}

1. Banaś, J, Goebel, K: Measures of Noncompactness in Banach Spaces. Lecture Notes in Pure and Appl. Math., vol. 60. Dekker, New York (1980)

2. Darbo, G: Punti uniti in trasformazioni a codominio noncompatto. Rend. Semin. Mat. Univ. Padova 24, 84-92 (1955)

3. Aghajani, A, Allahyari, R, Mursaleen, M: A generalization of Darbo's theorem with application to the solvability of systems of integral equations. J. Comput. Appl. Math. 260, 68-77 (2014)

4. Aghajani, A, Mursaleen, M, Shole Haghighi, A: Fixed point theorems for Meir-Keeler condensing operators via measure of noncompactness. Acta Math. Sci. 35(3), 552-566 (2015) 
5. Cai, L, Liang, J: New generalizations of Darbo's fixed point theorem. Fixed Point Theory Appl. 2015, Article ID 156 (2015)

6. Dhage, BC: Asymptotic stability of nonlinear functional integral equations via measures of non-compactness Commun. Appl. Nonlinear Anal. 15(2), 89-101 (2008)

7. Papageorgiou, SN, Kyritsi-Yiallourou, ST: Handbook of Applied Analysis. Springer Science \& Business Media, vol. 19. Springer, Berlin (2009)

8. Samadi, A, Ghaemi, MB: An extension of Darbo's theorem and its application. Abstr. Appl. Anal. 2014, Article ID 852324 (2014)

9. Samadi, A, Ghaemi, MB: An extension of Darbo fixed point theorem and its applications to coupled fixed point and integral equations. Filomat 28(4), 879-886 (2014)

10. Banaś, J, Nalepa, R: On a measure of noncompactness in the space of functions with tempered increments. J. Math. Anal. Appl. 435, 1634-1651 (2016)

11. Banaś, J, Mursaleen, M: Sequences Spaces and Measures of Noncompactness with Applications to Differential and Integral Equations. Springer, New Delhi (2014)

12. Banaś, J, Rodriguez, JR, Sadarangani, K: On a class of Urysohn-Stieltjes quadratic integral equations and their applications. J. Comput. Appl. Math. 113, 35-50 (2000)

13. Banaś, J, Sadarangani, K: Solvability of Volterra-Stieltjes operator-integral equations and their applications. Comput. Math. Appl. 41, 1535-1544 (2001)

14. Darwish, MA: On quadratic integral equation of fractional orders. J. Math. Anal. Appl. 311, 112-119 (2005)

15. Darwish, MA, Henderson, J, O'Regan, D: Existence and asymptotic stability of solutions of a perturbed fractional functional-integral equation with linear modification of the argument. Bull. Korean Math. Soc. 48, 539-553 (2011)

\section{Submit your manuscript to a SpringerOpen ${ }^{\circ}$ journal and benefit from:}

- Convenient online submission

Rigorous peer review

- Immediate publication on acceptance

- Open access: articles freely available online

- High visibility within the field

- Retaining the copyright to your article 